
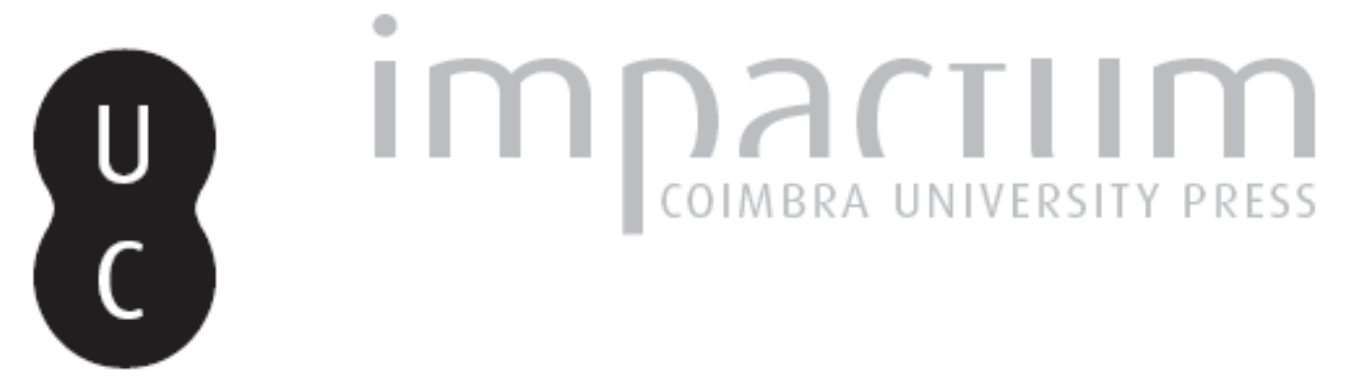

\title{
La «Guerre d'Elée» a-t-elle eu «lieu»?
}

\section{Autor(es): Nercam, Nathalie}

Publicado por: Imprensa da Universidade de Coimbra

URL persistente:

URI:http://hdl.handle.net/10316.2/42168

DOI:

DOI:https://doi.org/10.14195/2183-4105_12_2

Accessed : $\quad$ 26-Apr-2023 11:31:24

A navegação consulta e descarregamento dos títulos inseridos nas Bibliotecas Digitais UC Digitalis, UC Pombalina e UC Impactum, pressupõem a aceitação plena e sem reservas dos Termos e Condições de Uso destas Bibliotecas Digitais, disponíveis em https://digitalis.uc.pt/pt-pt/termos.

Conforme exposto nos referidos Termos e Condições de Uso, o descarregamento de títulos de acesso restrito requer uma licença válida de autorização devendo o utilizador aceder ao(s) documento(s) a partir de um endereço de IP da instituição detentora da supramencionada licença.

Ao utilizador é apenas permitido o descarregamento para uso pessoal, pelo que o emprego do(s) título(s) descarregado(s) para outro fim, designadamente comercial, carece de autorização do respetivo autor ou editor da obra.

Na medida em que todas as obras da UC Digitalis se encontram protegidas pelo Código do Direito de Autor e Direitos Conexos e demais legislação aplicável, toda a cópia, parcial ou total, deste documento, nos casos em que é legalmente admitida, deverá conter ou fazer-se acompanhar por este aviso.

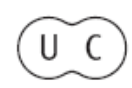


O

Established 1989

$\bigcirc$

$\vdash$

$<$

$\dashv$

ㅇ

-
$Z$
0
-
1
$Z$
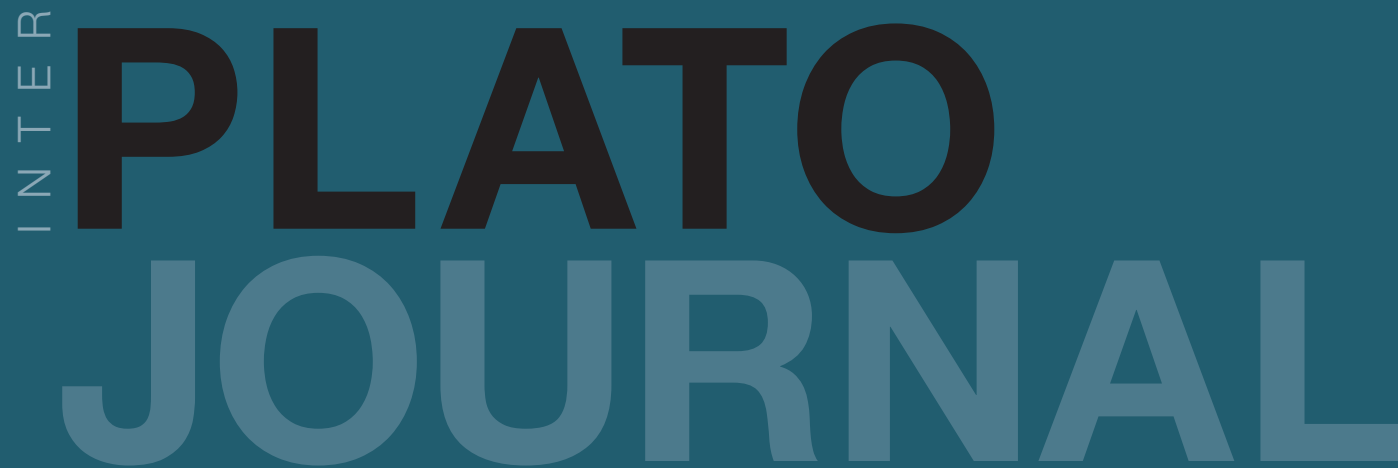

Société Platonicienne Internationale

Associazione Internazionale dei Platonisti

Sociedad Internacional de Platonistas

Internationale

Platon-Gesellschaft 


\title{
LA « GUERRE D’ELÉE » A-T-ELLE EU « LIEU » ?
}

\author{
Nathalie NERCAM
}

RESUME : Dans la première partie du Théétète, Socrate présente l'opposition entre pluralisme et monisme sous la forme métaphorique de la Guerre de Troie. Cette dramaturgie particulière permet à Platon de faire valoir une question philosophique qui proviendrait de Parménide. La mise en image platonicienne interroge en fait la réalité et le sens de khôra/topos.

ABSTRACT: In the first part of Theaetetus, Socrates shows the opposition between pluralism and monism through the metaphoric form of the Trojan War. This particular dramatization allows Plato to make evident a question inherited from Parmenides. This platonic picture questions the reality and the meaning of khôra/topos.

De façon consensuelle, l'exégèse contemporaine considère que Platon a survalorisé un aspect secondaire de la pensée de Parménide : l'unité de l'être. Le philosophe d'Elée ne serait pas le défenseur encore moins le promoteur du «monisme $»^{1}$. Jaap Mansfeld a montré que d'anciennes compilations issues probablement de la Première Sophistique avaient, avant Platon, classé les philosophes selon des critères particuliers et notamment en fonction du nombre de principes reconnus par chacun d'eux ${ }^{2}$. Il aurait donc été déjà admis au IV $^{\circ}$ siècle de présenter Parménide comme un tenant de l'unité. Nestor Cordero a précisé la façon dont Platon, dans le Sophiste, a adopté et transformé à ses propres fins, ces anciens schémas classificatoires ${ }^{3}$. On peut conduire une analyse semblable sur le Théétète, dialogue dans la première partie duquel Platon réemploie un classement d'origine très probablement doxographique dont Isocrate donne un exemple dans deux de ses discours. Cet article se propose de répondre à la double question: comment et pourquoi dans le Théétète, Platon a-t-il repris à son compte des classifications déjà établies avant lui ?

\section{La « guerre d'Elée »}

Par principe maïeutique, Socrate ne développe que des arguments appartenant au fond de connaissances de son jeune vis à vis. La démonstration du philosophe, dans la première partie du Théétète, pourrait donc reposer sur d'anciennes compilations connues de l'élève mathématicien. Or, comme l'a fait

\footnotetext{
${ }^{1}$ Voir en particulier BARNES, 1979.

${ }^{2}$ MANSFELD, 1990.

${ }^{3}$ CORDERO, 1991.
}

PLATO, The electronic Journal of the International Plato Society, 12, 2012. http://gramata.univ-paris1.fr/Plato/article116.html,

(c) All rights of reproduction of any form reserved. 
remarquer Jaap Mansfeld, un classement des philosophes est proposée par Isocrate dans le discours Sur l'échange où les théories présocratiques sont distribuées en fonction du nombre de réalités en chaque cas prises en compte ${ }^{4}$. Isocrate déclare: «l'un prétendait que les êtres sont en nombre infini, tandis qu'Empédocle n'en voyait que quatre avec la discorde et l'amour entre eux, Ion n'en comptait pas plus de trois, Alcméon deux seulement, Parménide et Melissos un, Gorgias absolument aucun $\gg^{5}$. Le rhéteur avait déjà utilisé le même principe classificatoire dans l'Eloge d'Hélène. «Comment surpasserait-on, écrivait-il, Gorgias qui osa déclarer que rien n'existe de ce qui est, ou Zénon qui tente de présenter successivement la même thèse comme possible ou impossible, ou Melissos qui devant la masse infinie des réalités existantes s'efforça de découvrir une preuve que leur ensemble formait un tout unique ? $\gg^{6}$.

Platon dans la première partie du Théétète a adopté un classement semblable sur son principe à celui d'Isocrate: les anciens philosophes sont ordonnés en fonction du nombre de leurs principes. Mais si le rhéteur présente les théories philosophiques en leur diversité, il en va tout autrement dans l'exposé socratique ${ }^{7}$. En effet les penseurs grecs évoqués sont tous regroupés en deux ligues opposées. D’un côté, Héraclite, Empédocle, Protagoras et Epicharme sont assemblés en un vaste dispositif dont Homère est le stratège. Ils affirment tous d'une seule voix, la pluralité et la mobilité de toute réalité (152e2-7 et 180d8) et font le siège d'une citadelle défendue par «les Melissos » et «les Parménide » qui proclament au contraire l'unité et l'immobilité de l'être (180e2-4). La classification doxographique aurait ainsi été simplifiée, réduite en une confrontation schématique entre défenseurs de la thèse de «l'unité statique » et défenseurs de l'antithèse, « la multiplicité changeante ».

Isocrate cherchait à exposer en quelques lignes les principes qu'il attribuait aux divers philosophes. Le Socrate de Platon propose au jeune Théétète une métaphore poursuivie sur plus de vingt pages. Il donne en effet à l'opposition des thèses la forme de l'affrontement guerrier. Ainsi Parménide, seul face à l'armée des pluralistes (152e2) est semblable au héros troyen Priam (183e5). En 180e1-2, Socrate cite un vers du Poème pour illustrer le parti pris «des Melissos» et «des Parménide » qui combattent les « Homériques ». La métaphore de la guerre est développée au delà de ces trois allusions directes. En 165d, la discussion dans laquelle s'engage le jeune Théétète n'est qu'un « combat de parole » et en $179 \mathrm{~d} 4$, le débat engagé autour de la thèse mobiliste est à nouveau considéré comme une « bataille ». Ce leitmotiv guerrier est par ailleurs diversement décliné : le conflit est aussi compétition chorale (179d7) puis jeu comme dans les palestres (181a2). En tous ces cas, le combat, l'agôn, est ouvert

\footnotetext{
${ }^{4}$ MANSFELD, 1990, p : 26.

${ }^{5}$ Sur l'échange, 268.

${ }^{6}$ Eloge d'Hélène, 3.

${ }^{7}$ Entre 152 e et 183 e.
}

PLATO, The electronic Journal of the International Plato Society, 12, 2012. http://gramata.univ-paris1.fr/Plato/article116.html,

(c) All rights of reproduction of any form reserved. 
sous différentes formes, toutes consacrées par la Cité. Socrate ne décrit donc que la rivalité des théories entre elles et inscrit Parménide dans le champ de bataille des discours.

Isocrate caractérisait de façon lapidaire la réflexion de chaque philosophe. Dans le Théétète, lorsque Socrate désire percer la spécificité des vues d'un penseur particulier, en l'occurrence Protagoras, la métaphore du champ de bataille est abandonnée afin que le grand sophiste puisse se défendre en personne. Une apologie est alors longuement développée (165e7-168c5). S'il n'en va pas de même pour les défenseurs du camp opposé c'est parce que Socrate déclare explicitement ne pas disposer d'assez de temps pour examiner rigoureusement les positions de Melissos et de Parménide dont la complexité est à cette occasion soulignée (183e3-184b2). Les deux thèses monolithiques qui s'entrechoquent n'appartiennent donc à aucun individu singulier. Le conflit est celui des représentations idéologiques, images des idées philosophiques réduites en affirmations massives et partagées en deux camps schématiquement opposés.

Isocrate appréciait les théories philosophiques selon un même critère, le nombre de réalités. Elles étaient ainsi placées comme à équivalence en un rapide tour d'horizon. La dimension guerrière donnée au conflit dans le Théétète implique au contraire un camp vainqueur et un autre vaincu. Les slogans ne sont donc pas à égalité. De plus, la fresque épique est dépeinte ouvertement à la façon d'Homère, comme une nouvelle guerre de Troie. Or c'est justement le grand Aède qui dirige les troupes des «pluralistes/mobilistes». La restitution métaphorique du combat serait donc de parti-pris. Platon ferait fonds sur un cercle historiographique bien connu : si guerre il y a, elle est toujours décrite par ceux qui en sont sortis vivants donc vainqueurs. En usant des mots et des images appartenant au camp des « Homériques », Socrate indiquerait ainsi que la thèse de ces derniers en l'emportant sur celle des « Eléatiques » constitue, à l'époque du jeune Théétète, la représentation idéologique dominante qui détermine le cadre et les termes du débat et qui définit en particulier les belligérants. Socrate aurait donc adopté le point de vue des «pluralistes » pour présenter la tendance « Parménide », vaincue sur le plan de l'opinion, cependant qu'en parallèle, dans le même ouvrage, il aurait produit une critique raisonnée de la thèse «pluraliste » dominante, mais défaite finalement par la démonstration. Dans ce jeu métaphorique, Platon croise donc les arguments. C'est ainsi qu'il use du principe unitaire pour assembler les «pluralistes » sous une même bannière. Ils triompheraient donc des «Eléatiques» dans «l'union», ce qui rend évidemment cette victoire très relative.

Après les avoir présentées, Isocrate disqualifiait les diverses thèses présocratiques, toutes finalement rejetées par le rhéteur au titre «d'artifice charlatanesque », les antiques penseurs affectant selon lui de confondre en paroles l'auditeur ${ }^{8}$. Pour Platon, ces vieilles querelles qui peuvent et doivent être

${ }^{8}$ Eloge d'Hélène, 4 .

PLATO, The electronic Journal of the International Plato Society, 12, 2012.

http://gramata.univ-paris1.fr/Plato/article116.html,

(C) All rights of reproduction of any form reserved. 
dépassées, ne sont pas des jeux artificieux. Elles prennent même, sous couvert d'ironie et par métaphore interposée, une ampleur littéralement épique, comme s'il s'agissait d'un choc de civilisations.

Il convient d'ajouter enfin que la guerre de Troie est l'image de prédilection par laquelle les orateurs athéniens décrivent les conflits armés de leur temps ${ }^{9}$. Platon emprunterait donc à l'art oratoire, et le principe classificatoire, et la métaphore. Mais il appliquerait ces moyens à décrire un combat tout à fait singulier. Loin de présenter comme Isocrate le vaste panorama des anciennes thèses philosophiques sur le terrain neutre de sa page, Platon réduirait la multiplicité des théories à une simple opposition binaire dont la connotation guerrière transforme complètement l'appréhension. Ce duel polarise en effet tout le débat autour de la philosophie de Parménide. Si elle sort vaincu de l'épreuve du combat des opinions, on ne pourrait, selon Platon, avaliser cette défaite sans procéder à un examen sérieux des questions soulevées par l'ancien philosophe d'Elée.

\section{Khôra dans la «Guerre d'Elée ».}

Les enjeux principaux de cette guerre idéologique sont bien connus. La critique contemporaine reconnaît en général deux des mots d'ordre affichés sur les bannières du Théétète : pluralisme contre monisme, dynamisme contre statisme. La prise en compte du contexte épique dans lequel Platon situe ce débat, permet d'accorder à un troisième terme une importance que le seul examen des slogans ne permet pas toujours de soupçonner. Ce mot polémique est «khôra ».

Socrate résume en effet en une seule formule la position défendue par « les Melissos et les Parménide». Ils soutiennent que « tout est un et se tient immobile en soi-même, n'ayant point de place (khôra) en laquelle se mouvoir » (180e3-4). La position des «Eléatiques» étant inverse à celle des «Homériques », il suit que pour ceux-ci, tout est multiple, en mouvement perpétuel et qu'il existe une place nécessaire à ce changement comme à cette multiplicité. On peut vérifier l'importance de khôra dans la thèse «pluraliste/mobiliste », en examinant les trois autres occurrences de ce mot. Ce sont les seules du dialogue. Khôra n'est donc employée dans cet ouvrage que pour décrire la réalité selon les « Homériques ». A leurs yeux, rien n’a de localisation propre dans un flux généralisé et supposé a priori. La couleur par exemple n'est ni dans l'œil, ni dans l'objet. Elle n'a pas même de place ( $\chi \omega \dot{\omega} \rho \alpha \nu$, première occurrence en $153 \mathrm{e} 1$ ) où se ranger puisqu'elle est en mouvement permanent. Seules existent des forces actives et passives qui déterminent des sensations et des qualités. En 181 c6 et toujours par la voix de Socrate, les mêmes « Homériques » définissent le mouvement. Il consiste soit à se déplacer d'une « place à une autre » $(\chi \omega \dot{\omega} \rho \alpha \nu$ ह่ $x$ $\chi \omega \dot{\omega} \rho a \zeta$, deuxième et troisième occurrences en $181 c 6$ et $\left.c 6^{2}\right)$ soit à tourner sur soi-

${ }^{9}$ Voir en particulier Isocrate : Panégyrique, 81-84, Eloge d'Hélène, 67.

PLATO, The electronic Journal of the International Plato Society, 12, 2012.

http://gramata.univ-paris1.fr/Plato/article116.html,

(C) All rights of reproduction of any form reserved. 
même, soit enfin à s'altérer. Les trois types de changement se cumulant, la réalité est toujours dans un mouvement perpétuel et général. Selon les «Homériques », tout est donc en changement, en particulier de place en place (181c6) donc rien n'a de place assignée (153e1). Khôra serait en quelque sorte le support général de ces dynamiques dans quoi ou par quoi de multiples réalités se meuvent et s'altèrent. Elle pourrait à ce titre faire partie des grands thèmes du « combat de parole » engagée entre les « Eléatiques » et les « Homériques ».

En considérant la métaphore par laquelle Socrate présente le siège d'Elée, on peut confirmer l'hypothèse précédente et préciser quelques uns des caractères de cette problématique khôra. Remarquons tout d'abord que le mot appartient au vocabulaire de l'Iliade et de l'Odyssée et signifie l'espace de terre limité et occupé par quelqu'un ou quelque chose. Or le conflit des représentations est par image interposée, situé précisément devant la citadelle d'Elée, la nouvelle Troie. Il prend donc littéralement « place ». Cette khôra métaphorique, la cité «troyenneéléate », est un singulier champ de bataille.

En effet aucun repère, ni chronologique, ni géographique n'est fixé car l'exposé de Socrate est bâti en dépit de toutes les distances. Protagoras d'Abdère côtoie Héraclite d'Ephèse, voisin d'Empédocle d'Agrigente et d'Epicharme de Syracuse. Ces divers personnages sont tous réunis en Asie Mineure devant la cité de Troie. Mais comme celle-ci représente Elée qui se trouve en grande Grèce, la scène se déroule à la fois à l'est et à l'ouest d'Athènes. La «place » que tient victorieusement l'armée homérique est donc multiple, éclatée dans les temps (d'Homère à Protagoras) et dans les lieux (des quatre coins de la Grèce). Ni l'ordre historique ni l'ordre géographique n'organisent cette mêlée dans laquelle espace et temps semblent confondus. Platon situe donc métaphoriquement le conflit dans une khôra proprement «mythologique» c'est-à-dire d'avant Hécatée, Hérodote et Thucydide.

Cette métaphore est riche d'enseignement car elle vaut comme illustration de ce qui est par ailleurs déclaré sur les bannières. Ainsi lorsque les «pluralistes/mobilistes » présupposent khôra, le lecteur est aussitôt renvoyé à cette étrange confusion dans laquelle Socrate place justement l'armée homérique par le moyen de l'image. La correspondance est cohérente. En effet, selon le principe défendu par les «pluralistes/mobilistes », khôra (comme toute chose) ne peut posséder aucune unité pas même sémantique et en conséquence, ne désigne qu'une réalité confuse, qu'illustre éloquemment le champ de bataille brossé par Socrate, mêlée des forces, des temps et des lieux. Parallèlement, les «Eléatiques » réfutent la multiplicité et le changement et n'accordent aucune réalité à ce qui est nommé khôra. Ce dernier point s'explique aisément si l'on considère la «place » métaphoriquement tenue par les « Homériques » et qui est précisément multiple donc sans réalité aux yeux des « Eléatiques ».

Le procédé de Platon qui prête à Socrate un savant double jeu, entre le sens des propositions et leur mise en forme littéraire, implique deux interrogations. Premièrement, la réalité dénotée par khôra peut être mise en question : par ce mot, la langue commune viserait non point un espace clairement

PLATO, The electronic Journal of the International Plato Society, 12, 2012.

http://gramata.univ-paris1.fr/Plato/article116.html,

(C) All rights of reproduction of any form reserved. 
distingué mais une unité syncrétique fusionnant notamment les dimensions temporelle et spatiale. Mais deuxièmement, la manière de dire la réalité est elle aussi en question. Car c'est l'art littéraire de Platon qui donne à khôra comme une patine archaïque. Le lieu illustré par la métaphore est à la fois une trouble réalité physique et une artificielle image rhétorique, ce qui met ainsi en question le rapport entre la(les) réalité(s) et la(les) manière(s) d'en parler.

La critique des «Eléatiques » serait alors et en quelque sorte soutenue par l'exemple, khôra restant dans le texte une notion problématique qui ne peut être abordée que par un incessant et implicite aller retour entre ce qui est dit et la manière de le dire. Mais la pure et simple négation de khôra, à défaut de diacritique et sans solution alternative, est insuffisante et donc perdante, sur le plan des représentations. Les défenseurs de l'«Eléatisme» seraient en effet contraints soit à se retirer d'un conflit dont ils n'acceptent pas le terrain et à être ainsi réduits à se taire, soit à participer au combat et ce faisant, à admettre d'être situés dans khôra. Platon présente ainsi la «vulgate éléatique » comme vaincue en quelque sorte dans et par khôra, terme à ce titre décisif dans le conflit des représentations.

\section{Topos, soumis à la critique.}

Les exégètes contemporains ont longuement débattu pour savoir si « l'un » était ou non le maître mot de la philosophie de Parménide mais n'ont jamais demandé ce qu'il en était au sujet de khôra. Les fragments du Poème permettent-ils de donner quelque réalité à la métaphore de Socrate ?

Le mot khôra n'y est jamais employé. Il existe par contre une occurrence de topos au vers $41 \mathrm{du}$ fragment VIII. Faut-il considérer qu'au $\mathrm{V}^{\text {ème }}$ siècle, les deux termes ont des sens différents? La réponse est négative de l'avis unanime des critiques $^{10}$. Ces deux mots désigneraient la situation relative où une chose se trouve comme le lieu particulier qu'elle occupe, topos renvoyant en général à un espace plus restreint que khôra. Tous les traducteurs du Poème s'accordent ainsi à donner à topos un sens globalement spatial. Il est le plus souvent traduit en français par «lieu $»^{11}$, parfois par «place $»^{12}$. Aucun commentaire ne venant préciser ces traductions, elles relèveraient du sens commun et en réferreraient en conséquence à une dimension d'espace assez floue et sans technicité particulière.

Considérons plus précisément le vers VIII-41 pour déterminer s'il présente ou non quelque analogie avec la position critique prêtée par Socrate aux

\footnotetext{
${ }^{10}$ Entre ces deux termes, écrit Luc Brisson, « la distinction n'existait pas, du moins pas à un autre niveau qu'à celui, plein d'imprécision du vocabulaire quotidien » (BRISSON, 1974, p : 213).

11 COULOUBARITSIS, 1986 ; CASSIN, 1998; BOLLACK, 2006; CONCHE, 1996 ; BEAUFFRET, 1955.

${ }^{12}$ CORDERO, 1984; O'BRIEN/FRERE, 1987. «Place » encore en anglais par exemple pour COXON, 2009 et GALLOP, 1984.
}

PLATO, The electronic Journal of the International Plato Society, 12, 2012.

http://gramata.univ-paris1.fr/Plato/article116.html,

(C) All rights of reproduction of any form reserved. 
«Eléatiques» dans le Théétete. La Déesse déclare que les mortels sont convaincus : « de naître et périr, être et ne pas être, changer de lieu <topos> et varier d'intensité lumineuse $\gg^{13}$.

Comme dans le Théétète, les opinions et les représentations dominantes transcrites dans la langue du mythe sont en question. Elles sont critiquées par la Déesse qui déclare en effet que les humains qui nomment les réalités s'égarent loin de la vérité. Cette errance a pu être interprétée de deux façons, soit les mortels ratent l'être qu'ils visent en le nommant, soit ils se perdent dans le vide, donnant des noms sans aucune réalité référentielle ${ }^{14}$. La critique de la Déesse s'exerce clairement à l'encontre de tous les noms et en particulier contre ceux des vers 40 et 41. En conséquence soit le sens de topos échappe aux humains, soit le mot luimême est absurde et vide de toute signification. Dans tous les cas, topos est flétri par la critique de Parménide, tout comme l'est khôra par les «Eléatiques » dans le Théétète.

Le changement est le thème principal du vers en question. Deux verbes l'expriment : $\dot{\alpha} \lambda \lambda \dot{\alpha} \sigma \sigma \varepsilon \iota \nu$ et $\dot{\alpha} \mu \varepsilon i \beta \varepsilon \iota \nu$ qui signifient «changer » et «échanger » dans le sens de « remplacer » ou « succéder ». Dans la formule de la Divinité, il peut y avoir altération (le lieu change) comme déplacement (changer d'un lieu pour un autre). Ces changements dans l'espace sont reliés aux variations lumineuses. Parallèlement, entre «naître» et «périr», « être» et « ne pas être », il y a encore «changement » et «échange » d'un terme positif à son contraire. Vivre serait ainsi selon les mortels : changer de lieu, dans des lieux euxmêmes changeant, passer du sein maternel à la lumière du jour puis de celle-ci à la pénombre de l'Hadès, transiter du néant à l'être et inversement de l'être au néant. Dans le vers VIII-41 du Poème, topos est donc associé étroitement au changement sous de multiples formes, comme l'est khôra dans le Théétète.

Les deux vers sont en leur forme marqués par un jeu d'assonances, de symétrie et de balancement à l'intérieur de chacun d'eux comme dans leur ensemble. Ces effets rhétoriques ajoutent au trouble conceptuel dénoncé par la Déesse. Ainsi comme dans le Théétète, c'est entre la réalité et la façon dont on peut la décrire que se noue le problème des mortels.

Notons enfin que dans ces multiples variations, le temps est toujours occulté tout en étant impliqué ; temps saisonnier qui fait varier la luminosité et les lieux, temps météorologique qui modifie tout l'environnement à un moment donné, temps enfin, nécessaire de manière générale à tout changement. Remarquons que le temps grec est mesuré très concrètement par le cadran solaire

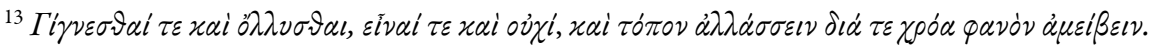

${ }^{14}$ Dans l'interprétation la plus fréquente, comme par exemple celle de Denis O’Brien et de Jean Frère, la dénomination humaine est vide de sens. Mais à partir de l'analyse d'une citation du Poème tirée du Théétète, Monique Dixsaut a proposé une lecture alternative : la dénomination des mortels vise la réalité mais la manque. Le nom n'est pas complètement vide, il désigne quelque chose. Mais il demeure cependant insensé, la chose étant comprise de façon ambiguë (O’BRIEN/FRERE, 1987 ; DIXSAUT, 1987).
}

PLATO, The electronic Journal of the International Plato Society, 12, 2012.

http://gramata.univ-paris1.fr/Plato/article116.html,

(C) All rights of reproduction of any form reserved. 
et le gnomon. Il est ainsi constaté de visu et in situ sur une surface éclairée. Dans ce dispositif, dominent lieu et lumière. Le vers 41 en réfererait ainsi indirectement à cette détermination particulière du temps.

La Déesse critique donc «topos » qui comme le mot «khôra » renvoie globalement pour l'opinion grecque à la localisation des choses. Elle l'associe étroitement à la notion de changement, tout en le dotant d'une dimension temporelle implicite mais déterminante. Elle fait enfin entendre qu'il existe de fait un rapport entre la réalité que l'on vise et la façon dont on en parle. Sur tous ces points, Platon dans le Théétete semble avoir assez scrupuleusement retenu la lettre et l'esprit du Poème. Khôra niée par les « Eléatiques » est sœur jumelle de topos disqualifié dans l'œuvre du philosophe d'Elée.

L'importance de cette critique aux yeux de Platon peut être complètement confirmée en examinant les lignes 172c2-177c6 qui forment le passage central de la première partie du Théétète dans laquelle Socrate présente « la Guerre d'Elée ». Le philosophe y abandonne temporairement le récit épique pour présenter deux paradigmes antagonistes qui illustrent deux types d'homme, d'une part le rhéteur tourné vers l'enquête judiciaire et d'autre part le physicien tourné vers l'examen du cosmos. Socrate fait ensuite valoir sa propre dynamique, celle de l'amélioration de soi, qui implique une orientation particulière vers la recherche du juste par l'imitation du divin. Dans tout ce passage, le mot khôra n'est pas employé. Mais le philosophe déclare vouloir fuir «le lieu <topos> d'ici » dans lequel le mal parcourt fatalement sa ronde (176a6-7). La critique de Parménide aurait donc porté; Platon semble ici l'enregistrer presque littéralement en lui donnant toutefois la saveur particulière qui est celle de la philosophie socratique. Qu'est ce en effet que le «lieu d'ici »? Il est lié à la nature mortelle et localise la sarabande des maux. Dans les versions françaises, il est traduit par « lieu d'ici-bas » ou « cette terre » ou encore «ce lieu-ci » ${ }^{15}$. Il renvoie ainsi à une réalité spatiale très générale, là où se trouve l'humaine nature et de ce point de vue en réfere directement au mot topos critiqué par la Déesse du Poème. Socrate cherche à s'en évader (176a-b). Or la Déesse ordonnait à Parménide de s'écarter du chemin qui est celui de l'opinion des mortels ${ }^{16}$. L'injonction était impérieuse. Le point de vue de Socrate n'est pas aussi autoritaire. Mais sur le fond, le philosophe d'Athènes obéit scrupuleusement à la consigne de la Divinité. Comme Parménide, le Socrate de Platon se détourne donc de topos. Mais il ajoute aussitôt chercher un « lieu <topos> pur de tout mal » qui semble comme au delà de la mort (177a5). Voilà qui dépasse de beaucoup la critique de Parménide mais qui est par contre précisément inscrit dans la dynamique socratique du Théétète. En effet comme on l'a précédemment constaté, en niant khôra, sans proposer d'alternative, les « Eléatiques » seraient enfermés dans le cercle de la métaphore guerrière. Pour sortir de cette impasse, il

\footnotetext{
${ }^{15}$ Successivement : DIES, 1926 ; CHAMBRY, 1967 ; NARCY, 1994.

${ }^{16}$ Poème 27-28, I et 3-5, VI.
}

PLATO, The electronic Journal of the International Plato Society, 12, 2012.

http://gramata.univ-paris1.fr/Plato/article116.html,

(c) All rights of reproduction of any form reserved. 
faut dégager positivement une nouvelle perspective. C'est peut-être ce qu'ont pu faire les physiciens et les rhéteurs en proposant des façons particulières et nouvelles d'appréhender le lieu, cosmologique et mathématique dans le premier cas, rhétorique et oratoire dans le second. Le fait est certain pour Isocrate qui emploie à plusieurs reprises dans ses discours, le mot topos dans le sens de «thème » ou de «sujet», signification fort éloignée de la confusion spatiotemporelle de khôra ${ }^{17}$. Mais de ces évolutions, Platon ne dit rien explicitement. Il met par contre en valeur la figure de Socrate dont Isocrate fut justement l'élève. Le philosophe athénien semble ainsi dépasser d'un même envol, d'une part les «Eléatiques », en outrepassant la critique du Poème contre topos, et d'autre part les modèles du rhéteur et du physicien qu'il pose comme deux paradigmes relatifs. Au delà de Parménide, Socrate cherche un lieu, et ce lieu dit « pur de tout mal » est comme au delà des réflexions du rhéteur et du physicien, peut-être parce que ceux-ci s'ignorant l'un, l'autre, ne peuvent jamais donner sur la réalité qu'un point de vue borgne, le lieu étant pour l'un rhétorique, pour l'autre, physique.

Tous ces éléments confirment l'importance accordée par Platon à la critique parménidienne. Elle a été positivement prise en compte en son implication majeure : le rejet de la confuse entité khôra/topos qu'exprime Socrate lorsqu'il désire s'évader hors de « ce lieu ci ». Mais la critique de Parménide a été aussi dépassée, le philosophe athénien cherchant un « lieu pur de tout mal » qui serait à ce titre débarrassé de toute confusion et de tout conflit.

Dans la première partie du Théétète, Platon reprend donc à son compte des classifications philosophiques déjà établies dont on connaît des exemples par Isocrate. Mais il simplifie et métaphorise ce classement sous la forme d'un duel épique: «Homériques» contre «Eléatiques». La classification est ainsi polarisée autour de Parménide.

Cette mise en image de la doxographie permet de mettre en question le « lieu ». Car les défenseurs de « l'Eléatisme » font directement écho à la critique que Parménide prononce contre topos dans le Poème, en contestant explicitement khôra admise par leurs opposants et Platon donne un relief particulier à cette contestation en situant métaphoriquement le débat aux portes d'une citadelle troyenne-éléate. Cette image littéraire fabriquée de toutes pièces peut renvoyer à une confusion des temps et des espaces, réelle ou présupposée, et désigne à la fois le lieu stratégique, site du duel, et le lieu rhétorique, sujet de la discussion.

Mais l'art de la parole, ainsi requis pour présenter la complexité des divers problèmes posés par le «lieu » qu'il soit appelé khôra ou topos, serait, pour les

\footnotetext{
17 Jacques Brunschwig, dans son introduction aux Topiques, relevait qu'avant Aristote seul Isocrate avait employé topos dans le sens « de «thème » ou de « sujet » (cf. l'anglais topics) donnant à un développement oratoire sa ligne directrice »(BRUNSCHWIG, 1967). Voir : Phillipe,109; Panathénä̈que, 88 et 111 ; Eloge d'Hélène, 4 et 38.
}

PLATO, The electronic Journal of the International Plato Society, 12, 2012.

http://gramata.univ-paris1.fr/Plato/article116.html,

(C) All rights of reproduction of any form reserved. 
résoudre, insuffisant. En dernière instance seul le philosophe Socrate ouvre une perspective positive, au delà du conflit des représentations, vers un topos dit « pur de tout mal ».

Platon a donc sollicité les savants moyens du rhéteur pour faire du « lieu » sous ses multiples aspects, le sujet du débat c'est-à-dire, selon les propres termes d'Isocrate, le « lieu » du débat. Ainsi le «lieu » de la Guerre d'Elée, est justement le «lieu », enjeu du conflit. De cette étrange dialectique, seul Socrate aurait pu finalement démêler l'écheveau.

PLATO, The electronic Journal of the International Plato Society, 12, 2012.

(c) All rights of reproduction of any form reserved. 


\section{BiBLIOGRAPHIE}

ISOCRATE , Discours, établi et traduit par Georges MATHIEU et Emile BREMOND, Belles Lettres, Paris, 1929.

PLATON, Théétète, établi et traduit par Auguste DIES, Belles Lettres, Paris, 1926.

BARNES J., 1979, «Parmenides and the Eleatic One », p :1-21, Archiv für Geschichte der Philosophie, $\mathrm{N}^{\circ} 61$.

BEAUfFRET J., 1996, Parménide, le Poème, P.U.F., Paris (première édition 1955).

BOLLACK J., 2006, De l'étant au monde, Verdier, Paris.

BRISSON L., 1974, Le même et l'autre dans la structure ontologique du Timée de Platon, Klincksieck, Paris.

BRUNSCHWIG J., 1967, Topiques d'Aristote, Belles Lettres, Paris.

BURNYEAT M., 1990, The Theaetetus of Plato, with a translation of Plato's Theaetetus by M.J. LEVETT revised by Myles BURNYEAT, Hackett Publishing Company, Indianapolis/Cambridge.

CASSIN B., 1998, Parménide. Sur la nature ou sur l'étant, Seuil, Paris.

CHAMBRY E., 1967, Platon, Théétète, Flammarion, Paris.

CONCHE M., 1996, Parménide, le Poème : fragments, P.U.F., Paris.

COULOUBARITSIS L., 1990, Mythe et philosophie chez Parménide, Ousia, Bruxelles (première édition 1986).

CORDERO N., 1997, Les deux chemins de Parménide, Vrin/Ousia, Paris/Bruxelles (première édition 1984).

1991, « L'invention de l'école éléatique, Platon Sophiste 242d », p :93-124, Etudes sur le Sophiste de Platon, sous la direction de Pierre AUBENQUE, Bibliopolis, Napoli.

COXON A.H., 2009, The Fragments of Parmenides, revised and expanded edition, new translations by R. McKIRAHAN and a new preface by M. SCHOFIELD, Parmenides Publishing, Las Vegas/Zurich/Athens.

DIXSAUT M., 1987, « Platon et le logos de Parménide », p : 215-253, Etudes sur Parménide, sous la direction de Pierre AUBENQUE, Vrin, Paris.

GALLOP D., 1984, Parmenides of Elea, University of Toronto, Toronto.

MANSFELD J., 1990, « Aristotle, Plato, and Preplatonic Doxography and Chronography », p : 22-83, Studies in the Historiography of Greek Philosophy, Van Gorcum, Maastricht, (première parution de l'article 1986).

NARCY M., 1994, Platon, Théétète, Flammarion, Paris.

O'BRIEN D. et FRERE J., 1987, Etudes sur Parménide, tome I, sous la direction de Pierre AUBENQUE, Vrin, Paris.

PLATO, The electronic Journal of the International Plato Society, 12, 2012.

http://gramata.univ-paris1.fr/Plato/article116.html,

(c) All rights of reproduction of any form reserved. 